\title{
Exploring Microsatellite Instability (MSI) in Colorectal Cancer at Elevated Microsatellite Alterations at Selected Tetranucleotides (EMAST)
}

\author{
Vadim Kurbatov, MD, MHS, and Sajid A. Khan, MD, FACS \\ Section of Surgical Oncology, Department of Surgery, Yale School of Medicine, New Haven, CT
}

Watson et al. demonstrate an increased frequency of elevated microsatellite alterations at selected tetranucleotides (EMAST) in elderly colorectal cancer (CRC) patients. ${ }^{1}$ EMAST is distinctly associated with frailty markers such as lower body mass index, hypoalbuminemia, anemia and higher CRPs, in addition to advanced age and improved recurrence-free survival (RFS) after surgical resection.

EMAST and high-frequency microsatellite instability (MSI-H) are often contemporaneous. MSI-H is one of the 2 major pathways of genomic instability in CRC, accounting for $20 \%$ of cases. ${ }^{2}$ It is characterized by diploidy and mutation or methylation silencing of mismatch repair (MMR) genes, resulting in instability at select mono- and dinucleotide loci (BAT25, BAT26, DSS123, D5S346, D17S250), which have defined MSI-H tumors since a 1997 National Cancer Institute (NCI) workshop. ${ }^{3}$ Technological advances in the ensuing decades have allowed for discovery and analysis of unique loci, such as tetranucleotide repeats, and new tumor genetic subtypes with clinically correlative features, presenting challenges of effectively incorporating these new understandings into clinical practice. ${ }^{4}$ Achievements like the comprehensive molecular characterization of CRC by The Cancer Genome Atlas

\footnotetext{
(C) Society of Surgical Oncology 2019
}

First Received: 17 September 2019;

Published Online: 1 December 2019

S. A. Khan, MD, FACS

e-mail: sajid.khan@yale.edu
(TCGA) project serve as important proof of this concept. ${ }^{5}$ The description of EMAST in CRC invites re-evaluation of the NCI workshop dogma in this context.

As the global population ages and life expectancy is forecasted to increase in nearly $60 \%$ of countries and territories by 2040 , more patients inevitably will become candidates for surgery. ${ }^{6}$ A clearer understanding of tumor biology can help inform surgical decision making in elderly CRC patients, such as Watson et al.'s clear demonstration of an association of the EMAST genotype with favorable surgical outcomes. Surgeons, who are understandably reluctant to offer curative intent surgery to older, frailer, patients because of increased perioperative risk, can consider surgery in the EMAST subset of elderly patients on account of favorable tumor biology with longer RFS. ${ }^{7}$ The inclusion of a high percentage of octogenarians speaks to the study strengths in providing a better understanding of tumor biology in this growing patient population. ${ }^{8}$

It is notable that nodal status was the strongest prognostic factor impacting RFS. In this context, we emphasize that the EMAST genotype should supplement traditional, well established clinical characteristics associated with outcomes such as nodal status. Additionally, since the phenotype associated with EMAST is characterized by advanced age and several frailty-associated characteristics, further clarification from the authors as to whether frailtyassociated characteristics are independently correlated with EMAST tumors or are correlating with advanced age would be useful. Finally, it would be interesting if the authors provided data on the frequency of tumor-infiltrating lymphocytes and impact of adjuvant systemic therapy on clinical outcome, since MSI-H tumors are sensitive to modern immunotherapy due to increased immunogenicity. ${ }^{9}$ 
The study by Watson et al. reminds us that by leveraging technologic advances of molecular biology, early models of cancer biology can be refined and extended. Harnessing this broadened understanding of tumor biology can help surgeons practice precision medicine to make decisions that maximize benefits and minimize risks.

DISCLOSURES The authors declare no conflict of interest.

\section{REFERENCES}

1. Watson M, Kanani A, Lea D, et al. Elevated microsatellite alterations at selected tetranucleotides in colorectal cancer is associated with an elderly, frail phenotype and improved recurrence-free survival. Ann. Surg. Oncol. 2019 (in press).

2. Grady WM, Carethers JM. Genomic and epigenetic instability in colorectal cancer pathogenesis. Gastroenterology. 2008;135(4): 1079-99.

3. Boland et al. A National Cancer Institute workshop on microsatellite instability for cancer detection and familial predisposition: development of international criteria for the determination of microsatellite instability in colorectal cancer. Cancer Res. 1998;58(22):5248-5257.
4. Nash GM, Gimbel M, Shia J, et al. Automated, multiplex assay for high-frequency microsatellite instability in colorectal cancer. $J$ Clin Oncol. 2003;21(16):3105-12.

5. Cancer Genome Atlas Network. Comprehensive molecular characterization of human colon and rectal cancer. Nature. 2012;487(7407):330-7.

6. Foreman KJ, Marquez N, Dolgert A, et al. Forecasting life expectancy, years of life lost, and all-cause and cause-specific mortality for 250 causes of death: reference and alternative scenarios for 2016-40 for 195 countries and territories. Lancet. 2018;392(10159):2052-90.

7. Turrentine FE, Wang H, Simpson VB, Jones RS. Surgical risk factors, morbidity, and mortality in elderly patients. J. Am. Coll. Surg. 2006;203(6):865-77.

8. Shenoy P, Harugeri A. Elderly patients' participation in clinical trials. Perspect. Clin. Res. 2015;6(4):184-9.

9. Ribic CM, Sargent DJ, Moore MJ, et al. Tumor microsatelliteinstability status as a predictor of benefit from fluorouracil-based adjuvant chemotherapy for colon cancer. $N$ Engl J Med. 2003;349:247-57.

Publisher's Note Springer Nature remains neutral with regard to jurisdictional claims in published maps and institutional affiliations. 